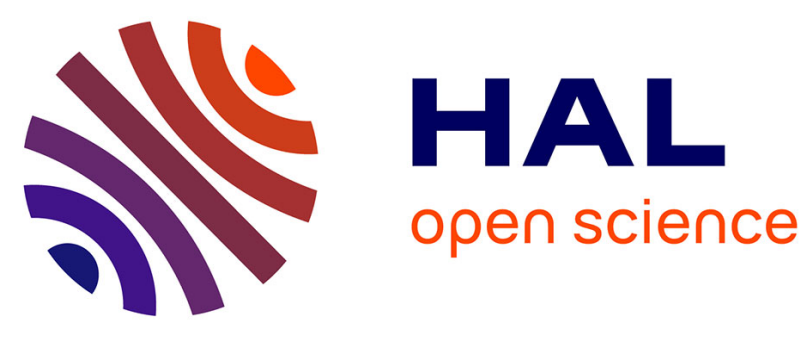

\title{
Emerging fields in chaperone proteins: A French workshop
}

Elisabetta Mileo, Marianne Ilbert, Alessandro Barducci, Patricia Bordes,

Marie-Pierre Castanie-Cornet, Cyrille Garnier, Pierre Genevaux, Reynald

Gillet, Pierre Goloubinoff, Françoise Ochsenbein, et al.

\section{To cite this version:}

Elisabetta Mileo, Marianne Ilbert, Alessandro Barducci, Patricia Bordes, Marie-Pierre CastanieCornet, et al.. Emerging fields in chaperone proteins: A French workshop. Biochimie, 2018, 151, pp.159-165. 10.1016/j.biochi.2018.06.004 . hal-01863220

\section{HAL Id: hal-01863220 \\ https://hal-univ-rennes1.archives-ouvertes.fr/hal-01863220}

Submitted on 3 Sep 2018

HAL is a multi-disciplinary open access archive for the deposit and dissemination of scientific research documents, whether they are published or not. The documents may come from teaching and research institutions in France or abroad, or from public or private research centers.
L'archive ouverte pluridisciplinaire HAL, est destinée au dépôt et à la diffusion de documents scientifiques de niveau recherche, publiés ou non, émanant des établissements d'enseignement et de recherche français ou étrangers, des laboratoires publics ou privés. 


\title{
Emerging fields in chaperone proteins: a French workshop
}

Elisabetta Mileo ${ }^{1}$, Marianne Ilbert ${ }^{1}$, Alessandro Barducci ${ }^{2}$, Patricia Bordes ${ }^{3}$, Marie-Pierre

Castanié-Cornet $^{3}$, Cyrille Garnier ${ }^{4,5}$, Pierre Genevaux ${ }^{3}$, Reynald Gillet ${ }^{6}$, Pierre Goloubinoff $^{7}$, Françoise Ochsenbein ${ }^{8}$, Gilbert Richarme ${ }^{9}$, Chantal Iobbi-Nivol ${ }^{1}$, Marie-Thérèse GiudiciOrticoni $^{1}$, Brigitte Gontero ${ }^{1}$, and Olivier Genest ${ }^{1}$

${ }^{1}$ Aix Marseille Univ, CNRS, BIP, Bioénergétique et Ingénierie des Protéines, Marseille, France.

${ }^{2}$ Centre de Biologie Structurales de Montpellier, CNRS UMR 5048 - UM - INSERM, Montpellier, France.

${ }^{3}$ Laboratoire de Microbiologie et Génétique Moléculaires, Centre de Biologie Intégrative, CNRS, Université Paul-Sabatier, Toulouse, France.

${ }^{4}$ Mécanismes Moléculaires dans les Démences Neurodégénératives, Université de Montpellier, EPHE, INSERM, U1198, F-34095 Montpellier, France and ${ }^{5}$ Université de Rennes 1

${ }^{6}$ Univ. Rennes, CNRS, Institut de Génétique et Développement de Rennes (IGDR) UMR6290, Rennes, France.

${ }^{7}$ Département de Biologie Moléculaire Végétale, Université de Lausanne, 1015 Lausanne, Suisse.

${ }^{8}$ Institute for Integrative Biology of the Cell (I2BC), Joliot, CEA, CNRS, Univ. Paris-Sud, Université ParisSaclay, Gif-sur-Yvette, France.

${ }^{9}$ UMR 8601 CNRS, Laboratoire de Chimie et Biochimie Pharmacologiques et Toxicologiques, Université Paris Descartes-Sorbonne Paris Cité, Paris.

\begin{abstract}
The "Bioénergétique et Ingénierie des Protéines (BIP)" laboratory, CNRS (France), organized its first French workshop on molecular chaperone proteins and protein folding in November 2017. The goal of this workshop was to gather scientists working in France on chaperone proteins and protein folding. This initiative was a great success with excellent talks and fruitful discussions. The highlights were on the description of unexpected functions and posttranslational regulation of known molecular chaperones (such as Hsp90, Hsp33, SecB, GroEL) and on state-of-the-art methods to tackle questions related to this theme, including Cryo-electron microscopy, Nuclear Magnetic Resonance (NMR), Electron Paramagnetic Resonance (EPR), simulation and modeling. We expect to organize a second workshop in two years that will include more scientists working in France in the chaperone field.
\end{abstract}

Keywords: Chaperone proteins, multifunctionality, plasticity, folding/misfolding, aggregation, structural dynamics. 


\section{Introduction}

The concept of molecular chaperones originates from the view that even though the structure (or absence of structure) of proteins is encoded by their primary amino acid sequence, sometimes they require "helper" proteins to reach their functional three-dimensional structure and avoid aggregation or degradation. The term of molecular chaperone was first coined to describe the function of a protein that assists the assembly of folded subunits into oligomeric protein complexes [1], and later was extended to protein folding [2]. A consensus now emerges to define molecular chaperones as a diverse group of proteins that assist the folding/unfolding of other proteins and the formation/dissociation of macromolecular complexes without being a component of the final complex. Molecular chaperones are involved in a wide range of biological processes and play key roles in all living organisms. They can be used or targeted for many medical, environmental or biotechnological applications.

At the end of November 2017, the Laboratory of "Bioénergétique et Ingénierie des Protéines" (BIP, Marseille) organized a workshop bringing together about fifty participants with guest speakers from France and one from Switzerland. Such an initiative had already been carried out in Europe, as for example in the United Kingdom (UK) and in the Netherlands. Meetings of this type are intended to improve communication within an emergent community and to increase collaborations and visibility at national and international levels.

In this report, we give an overview of the lectures (Figure 1). The first part focuses on the large panel of functions general chaperones may accomplish in various cellular processes under physiological as well as stressful conditions, showing an astonishing functional diversity. The two following sections concern chaperones that present particular features like intrinsic disorder and flexibility, and specific chaperones dedicated to a unique client. Here again, the diversity of the function of chaperones is illustrated. Finally, innovative techniques and tools to study or simulate chaperone behaviors that were exposed at the workshop are recorded in the last section of the report.

\section{Breakthrough in known chaperones}

General chaperones are proteins that maintain proteostasis in cells by preventing protein aggregation, helping in the native folding, activation and targeting of a large array of client proteins, in contrast to specific chaperones that are usually dedicated to a unique substrate. 
Some general chaperones require ATP for their function, whereas others are ATPindependent proteins. General chaperones have been extensively studied. Therefore, to continue making exciting breakthrough, original angles of research including unexplored model organisms and new chaperone discovery are needed.

\subsection{ATP and general chaperones}

Protein homeostasis in cells depends on energy-consuming processes. For instance, de novo protein synthesis requires ATP hydrolysis for peptide bond formation, and controlled degradation by the chaperone-gated proteases requires ATP hydrolysis to unfold target proteins and render their peptide bonds accessible to hydrolysis. During and following translation, different classes of molecular chaperones require ATP hydrolysis to control the conformational state of proteins and favor their folding into their active conformation. Furthermore, ATP-fueled unfolding chaperones, such as Hsp70, Hsp110 and Hsp104 can dynamically solubilize and reactivate stable protein aggregates that are formed under certain stress.

Pierre Goloubinoff (keynote lecture, Université de Lausanne, Switzerland) explained how he and his colleagues show, using various biochemical assays and physical modelling, that the highly conserved chaperones Hsp60 and Hsp70 by acting as ATP-powered unfolding enzymes, can inject the energy liberated by ATP-hydrolysis to destabilize misfolded polypeptides. By doing so iteratively at each ATP-cycle, these chaperones can artificially repopulate and transiently maintain the native state population of their substrate proteins, even under conditions that according to equilibrium thermodynamics, disfavor the native state. By acting as catalytic polypeptide unfolding nano-machines that specifically target misfolded and alternatively-folded protein substrates, chaperones may use the energy of ATP hydrolysis to reduce the cellular concentration of harmful non-functional protein aggregates and convert them into functional proteins [3].

Recent quantitative proteomic studies show that in metazoans, the main ATPase chaperones Hsp90, Hsp70, Hsp60, correspond to 3-4 \% of the total proteins of healthy terminally differentiated adult cells. Chaperones may be dramatically more abundant in healthy undifferentiated embryonic cells and in cancer cells, and much less abundant in aging and diseased tissues. For Pierre Goloubinoff, the future of chaperone research lies in the understanding of the fine-tune differences in chaperone and co-chaperone amounts and ratios in healthy embryonic cells and in cancer cells, both massively overexpressing chaperones, and 
diseased neuronal cells in degenerative diseases, like Parkinson and Alzheimer, that dramatically under-express chaperones and fail to repair or degrade toxic misfolded proteins.

\subsection{The Hsp90 chaperone}

The 90-kDalton heat-shock chaperone protein (Hsp90) has been well-studied in eukaryotes [4]. This three-domain protein is essential and allows in an ATP-dependent reaction the folding and activation of more than 300 client proteins with the assistance of about 20 cochaperones and the Hsp70 chaperone system. Among these clients, Hsp90 participates in the folding of oncoproteins in cancer cells. Therefore, many studies focused on Hsp90 inhibitor development to cure cancers. In addition, Hsp90 has been shown to regulate several amyloid proteins; it is thus considered as new target to treat amyloid diseases [5]. For instance, Hsp90 interacts with the amyloid protein tau. The interaction of Hsp90 with tau has been suggested to occur through tau aggregation-prone regions, including the VQIVYK motif. This hexapeptide Ac-VQIVYK-NH $\mathrm{NH}_{2}$ is responsible of tau amyloid character and is widely used as a model to study in vitro amyloid formation mechanisms; it is known to self-aggregate and to form amyloid fibrils [6]. Although it has been suggested that Hsp90 interacts with this hexapeptide, the role of the chaperone on the fibrillation process was still unknown. During his conference, Cyrille Garnier (MMDN Inserm, Montpellier) showed that Hsp90 (purified from pig brain) modulates in vitro the assembly and disassembly of amyloids structures formed from tau Ac-VQIVYK- $\mathrm{NH}_{2}$ hexapeptide. Hsp90 inhibited polymerization of the hexapeptide maintaining it in its soluble form. At high hexapeptide concentration when Hsp90 was "overbooked", polymerization occurred; Hsp90 interacted with mature fibrils preventing their depolymerization. This important function could limit the spread of toxic amyloid species. A model explaining the dual effect of Hsp90 on the Ac-VQIVYK- $\mathrm{NH}_{2}$ amyloid fibrillation process has been proposed [7]. Overall, this study gives insight into the roles of chaperone proteins in amyloid turnover.

In contrast to the great number of studies on eukaryotic Hsp90, Hsp90 from bacteria has been less investigated [8]. In Escherichia coli, Hsp90 is dispensable, few client proteins are known, and no co-chaperone has been described so far. Therefore, studying new bacterial model is crucial to characterize better prokaryotic Hsp90. Olivier Genest (CNRS, Marseille) presented experiments on the Hsp90 chaperone in Shewanella oneidensis (called Hsp90 ${ }_{\text {So}}$ ), an aquatic gamma proteobacterium that adapts to stressful conditions. He showed that $\mathrm{Hsp} 90_{\text {So }}$ is essential in $S$. oneidensis under heat stress, and that the ATPase activity of $\mathrm{Hsp} 90_{\text {So }}$ is 
required for this function [9]. To identify $\mathrm{Hsp} 90_{\text {So }}$ clients, a genetic screen using the growth phenotype at high temperature of a strain lacking Hsp90 $0_{\text {So }}$ was designed, and it allowed the identification of a protein involved in tRNA maturation, TilS. In the absence of $\mathrm{Hsp} 90_{\text {So }}$ and at sub-lethal temperature, a significant decrease in TilS activity was measured and TilS was degraded. Therefore, this study links the major role of Hsp90 in S. oneidensis under stress conditions with an essential client protein, and opens up new avenues to understand better the role and mechanism of action of the Hsp90 chaperone in bacteria.

\subsection{SecB-like chaperone}

In Proteobacteria, the SecB chaperone assists protein export and participates in the folding of certain cytosolic proteins [10]. Pierre Genevaux (CNRS, Toulouse) talked about the specialization of a SecB like chaperone in Mycobacterium tuberculosis and its link with toxinantitoxin (TA) systems. Bacterial type II toxin-antitoxin systems are small genetic modules typically composed of a toxin and a more labile cognate antitoxin, which binds and inhibits the toxin. In response to certain stress the antitoxin is rapidly degraded by proteases and the free active toxin generally targets essential cellular processes, leading to a reversible growth inhibition known to facilitate bacterial persistence to drugs, virulence and survival in response to environmental insults. Tripartite TAC (Toxin- $\underline{\text { Antitoxin- }}$ Chaperone) modules are atypical TA systems composed of a conserved two-component TA that is specifically controlled by a molecular chaperone related to the canonical SecB chaperone. In this case, the SecB-like chaperone interacts with the antitoxin and protects it from both aggregation and degradation, and is thus strictly required for neutralization of the toxin by the antitoxin. In his talk, Pierre Genevaux presented some aspects of the molecular mechanism by which TA systems become chaperone-addicted [11], the specialization events that redirect a generic export chaperone towards the control of TA systems [12] and some structural insights into the complex formed between the chaperone and the antitoxin. In addition, some part of the talk also concerned the direct evolution of the GroEL chaperone toward specific protein aggregates in E. coli (Castanié-Cornet et al., in preparation).

\subsection{Protein reparation}

Beside their role in protein folding, some chaperones allow the reparation of other proteins. Gilbert Richarme (keynote lecture, Université Paris Descartes-Sorbonne) reported that DJ1/Park7, whose deficiency results in early onset parkinsonism, and previously described as a chaperone, repairs proteins from glycation. Glycation is a non-enzymatic covalent reaction 
discovered by Maillard in 1912, between reactive carbonyls (glucose and glyoxals (R-CO$\mathrm{CHO})$ ), and amino acids (cysteine, arginine and lysine) or nucleotides (guanine), which results in the formation of Maillard adducts, followed by their maturation into the so-called advanced glycation end products (AGEs). Glycation is responsible for protein and nucleic acid inactivation, and is involved in aging, cancer, atherosclerosis, cataracts, neurovegetative, renal and autoimmune diseases. DJ-1 family proteins, named the "Maillard deglycases", are expressed in all organisms, and repair glycation by glyoxals, which represents about $70 \%$ of glycation damage [13]. Maillard deglycases also repair nucleic acids, especially the primary amino group of guanine, in a similar manner as they repair the lysine amino group [14]. Deglycases from thermophilic organisms may also be used to prevent AGEs and acrylamide formation during food processing sterilization and storage [15]. 


\section{Breakthrough in known chaperones}

Chaperones

Hsp90 Bacteria
New client involved in
tRNA modification

SecB $\longrightarrow \quad \begin{aligned} & \text { Bacteria } \\ & \text { Specialization to }\end{aligned}$ control toxin-antitoxin

Functions (discussed Eukaryotes

target for treatment of

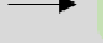

Reparation

Maillard

deglycases

$\longrightarrow \begin{gathered}\text { Reparation } \\ \text { of glycated } \\ \text { proteins and }\end{gathered}$ nucleic acids

Mechanism

Energy from

Hsp60

Hsp70

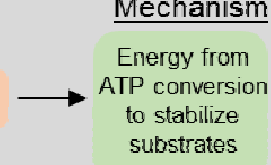

Specific chaperones

$1^{\text {st }}$ French Workshop

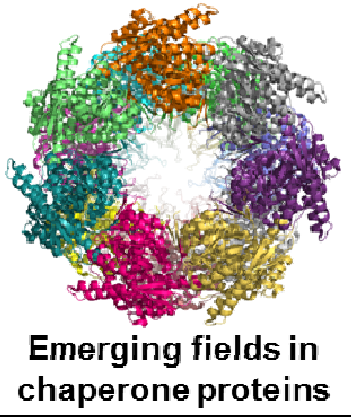

Amyloid fibril regulation

Plasticity in chaperone proteins

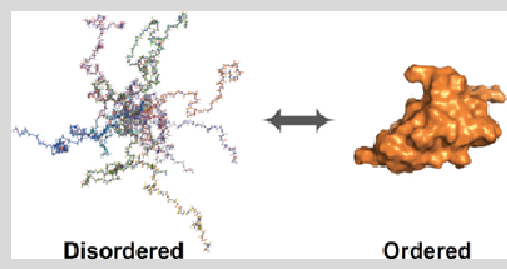

Reduced $\mathrm{CP} 12$

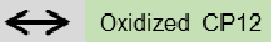

Oxidized Hsp33 $\longleftrightarrow$ Reduced Hsp33

Tools to study chaperone structural dynamics

« in-cell $»$ EPR spectroscopy

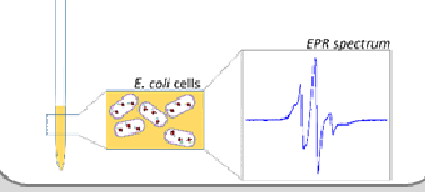

Molecular Dynamics Simulations

Chaperones

Functions

Eukaryotes

Asf1

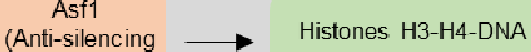

factor 1)

assembling

Bacteria

TorD family

Molybdoenzyme maturation
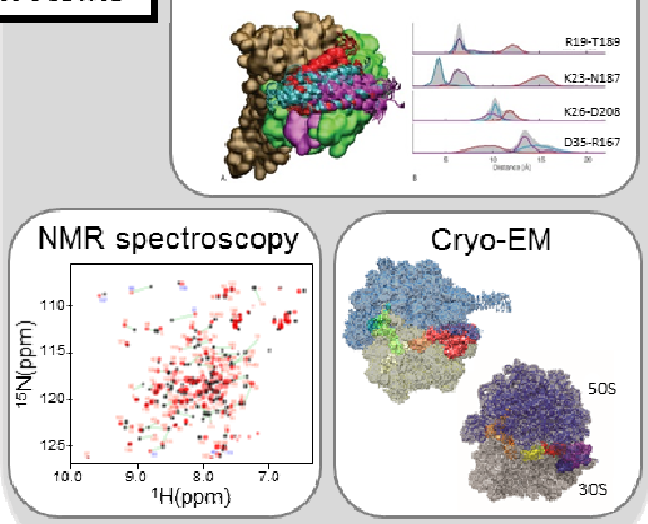

Figure 1: Overview of the topics discussed during the meeting. The scheme showing the disordered and ordered protein structures has been reprinted from reference [16] (Figure 1, Copyright (2018), with permission from Elsevier). In the panel "Tools to study chaperone structural dynamics", the NMR spectrum has been modified from reference [17], the Molecular Dynamics Simulations image has been reprinted from reference [18], the Cryo-EM maps have been reprinted from reference [19].

\section{Plasticity in chaperone proteins}

Some proteins challenge the classical structure-function paradigm because they do not have a well-defined three-dimensional structure. These proteins were originally termed intrinsically unstructured proteins (IUPs) and nowadays are called intrinsically disordered proteins (IDPs). 
Despite their lack of a well-defined three-dimensional structure, many proteins containing disordered regions/domains (IDRs) or completely disordered proteins are functional. These proteins carry out basic functions such as regulation of metabolic pathways, transcription, translation, cellular signal transduction; they can act as scavengers of toxic molecules and they play a key role in the assembly of multi-protein complexes. Moreover, recently they were shown to have other functional capacity as they can act as chaperones assisting the folding of other proteins and preventing their aggregation. For some proteins, a conformational switch between disordered and folded states or reciprocally, is redoxcontrolled by the formation/breakage of disulfide bonds (oxidation/reduction). These proteins belong to the conditionally disordered protein (CDP) family. Hsp33 and CP12 are two CDPs that both have chaperone activity [20].

\subsection{Hsp33 chaperone}

Marianne Ilbert (CNRS, Marseille) described the molecular mechanism of Hsp33, a molecular chaperone specifically activated by oxidative stress conditions that lead to protein unfolding. Exposure to peroxide stress at elevated temperature activates Hsp33. To respond to this dual stress, Hsp33 has developed a very ingenious system with (i) a C-terminal redox switch domain which detects oxidizing conditions by disulfide bond formation and (ii) an adjacent metastable linker region which responds to unfolding conditions [21]. Hsp33 is then turned into an active chaperone that protects the cells against oxidative protein aggregation. Use of a faster oxidative agent, hypochlorous acid $(\mathrm{HOCl})$ produced by the immune system in response to bacterial attacks, can activate the chaperone function of Hsp33 without the requirement of elevated temperature, underlying an even more interesting mode of regulation [22].

\subsection{CP12 protein}

Brigitte Gontero (CNRS, Marseille) presented a small chloroplast protein called CP12 found in most photosynthetic organisms [23]. In the green alga, Chlamydomonas reinhardtii, CP12 is constituted of about 80 amino acids and has two pairs of cysteine residues that under oxidizing conditions can form two disulfide bridges, one at the $\mathrm{N}$-terminus and one at the $\mathrm{C}$ terminus. Oxidized CP12 is present under dark conditions, and plays a vital role in the assembly of a complex involving phosphoribulokinase (PRK) and glyceraldehyde-3phosphate dehydrogenase (GAPDH), two enzymes belonging to the Calvin cycle responsible for $\mathrm{CO}_{2}$ assimilation. $\mathrm{CP} 12$ is required for the ternary complex assembly [24]. Its release from 
the complex is triggered by reducing conditions corresponding to light. Under these conditions, the ternary complex is dissociated, the released enzymes are fully active and reduced CP12 is fully disordered and highly flexible. CP12, besides its role in the assembly of the supra-molecular complex, can prevent the in vitro thermal inactivation and aggregation of GAPDH and as such has a chaperone activity [25].

\section{Specific chaperones}

During the workshop, a session was dedicated to specific chaperones. In contrast to a general chaperone that acts on a broad panel of client proteins, a specific chaperone is dedicated to a targeted client. During this workshop, two families of specific chaperones have been highlighted. One is dedicated to histones during the assembly process of chromatin, and the second is involved in the maturation of bacterial respiratory molybdoenzymes. These two examples show the large impact that specific chaperones possess in various cellular processes just as do general chaperones.

\subsection{Histone chaperones}

Chromatin assembly and disassembly processes involve a number of chaperoning factors playing crucial roles in the maintenance of the genome. Factors that assemble and remodel chromatin are fascinating machineries that not only act in the mechanical packaging of DNA but are also involved in the tight regulation of DNA accessibility in processes such as transcription, replication and repair. Francoise Ochsenbein (CEA, Gif-sur-Yvette) focused her talk on Asf1 (Anti-silencing function 1) a highly conserved histone chaperone that binds and assembles histones H3-H4 onto the DNA, and is required for cell proliferation, cell cycle progression, resistance to genotoxic stresses and the establishment of specific histone epigenetic marks [26]. During her talk, Francoise Ochsenbein exposed an innovative peptidic development on the interaction between Asf1 chaperone and H3-H4. This high-affinity peptide competes with Asf1 binding in vitro, and binds Asf1 in human cells. On cell cultures, the inhibitory peptide was found to be toxic for U2OS cancer cells impeding proliferation by slowing down the cell cycle progression but remained sensitive to proteolysis. Since a recent study showed a striking correlation between the severity of breast cancers and the level of Asf1 expression, this phenotype highlights the specificity of the peptide action and opens major perspectives in chaperone targeted therapies. 


\subsection{Chaperones of metalloenzymes}

Bacterial metalloenzymes and especially molybdoenzymes are fully involved in environmental purposes since they have crucial functions in respiration, detoxification processes and are part of various geochemical cycles. In bacteria, most of the molybdoenzymes require specific chaperones for their stability and maturation (i.e. insertion of a complex molybdenum cofactor) [27]. The knowledge acquired on both the function and the mechanism of these dedicated auxiliary proteins is of great applied interest. The molybdoenzyme TorA and its mate chaperone TorD have been studied in E. coli and $S$. oneidensis in particular by Marianne Ilbert, Olivier Genest, and Chantal Iobbi-Nivol (CNRS, Marseille). These studies allowed: (i) to define a family of specific chaperones for molybdoenzymes, the TorD like family; (ii) to demonstrate that the specific chaperone TorD prevents the degradation of the non-mature form of TorA by the Lon protease [28]; (iii) to understand the mechanism of insertion of the molybdenum cofactor into TorA [29].

Other studies performed on the membranous nitrate reductase NarGHI showed that its specific chaperone NarJ is also involved in the assembly of the three subunits and the anchoring of the enzymatic complex to the membrane. This chaperone protein has been the focus of another talk (see part 5). Altogether, studies performed on the various members of the TorD-like family highlight the wide panel of tasks undertaken by these specific chaperones and that they share a highly conserved 3D-structure although they present a low level of amino acid sequence homology [29].

\section{Experimental challenges and biophysical tools for studying chaperone structural dynamics and their interactions}

Protein structural flexibility and dynamics play a central role in biological functions. Indeed, there is an intimate relation between dynamics and molecular function. Structural transitions are involved in many key biological processes and play an important role in molecular recognition processes, catalysis, ligand binding, assembly of complex structures and disorderto-order transitions, as already mentioned for CP12 and Hsp33 (see part 3).

Chaperones are a fascinating group of proteins that exhibit different degrees of structural flexibility and are able to interact with different partners or different regions of the same partner thereby modulating their properties. Understanding chaperone dynamics and their interaction with other biomacromolecules is a key objective to decipher how they function 
and how their dynamics is related to their biological activity. However, due to the intrinsic dynamics and to the high structural fluctuations of chaperones, this kind of studies is currently difficult. During the workshop, several talks focused on the importance of biophysical approaches and on emerging experimental techniques in studying chaperone dynamics.

\subsection{Modeling}

Alessandro Barducci (INSERM, Montpellier) showed how the combination of analytical models, multi-scale molecular simulations and statistical analysis of protein sequences is a powerful approach to shed light on the complex functional cycle of Hsp70 chaperones. The ubiquitous Hsp70s are highly versatile, ATP-dependent molecular machines in the chaperone toolbox that assist a plethora of cellular processes including co-translational folding, refolding of misfolded and aggregated proteins, protein translocation, and protein degradation.

Particularly, A. Barducci explained how rate models based on biochemical rates can be used to quantitatively investigate the non-equilibrium cycling of Hsp70 chaperones, which take advantage of the energy released by ATP-hydrolysis to achieve ultra-affinity for the client proteins [30]. Furthermore, it was discussed how multi-scale molecular simulations can be profitably combined with co-evolutionary sequence analysis to characterize large-scale conformational dynamics and homo-dimerization in Hsp70 [31], as well as to elucidate key structural features of the transient complex formed by this chaperone with essential cochaperones, such as J-domain proteins [18].

\subsection{Cryo-electron microscopy}

In bacteria, trans-translation is the primary quality control mechanism for rescuing bacterial ribosomes arrested during translation. This key process is universally conserved and plays a critical role in the viability and virulence of many pathogens. Taking into account the necessity for bacterial survival to avoid ribosome stalling and the absence of trans-translation in eukaryotes, this process is a promising target for novel antibiotics or for increasing the activity of currently used protein synthesis inhibitors. Reynald Gillet (Université de Rennes) presented recent results obtained on structural aspects of trans-translation using cryo-electron microscopy, addressing the movements of the complex between transfer-messenger RNA (tmRNA) and SmpB protein (tmRNA-SmpB) through a stalled ribosome, and more particularly the conformational changes needed to transit from one ribosomal site to another [32]. The data obtained from this study were used to develop an in vivo double fluorescent 
reporter system for simultaneous quantification of trans-translation and associated proteolysis activities in bacteria [33]. This assay can be used to discover potent inhibitors of transtranslation in vivo.

\subsection{Magnetic Resonance Studies}

Françoise Ochsenbein (CEA, Gif-sur-Yvette), whose work has been already introduced (see part 4), showed the importance of integrating NMR spectroscopy, X-ray crystallography and modeling methods to characterize the structure of protein complexes. In particular, she highlighted how the characterization and the understanding of the interaction network between Asf1 histone chaperone and histones H3-H4 was possible thanks to this integrative biophysical approach [34,35].

Concerning the "emerging experimental techniques", the study of biomolecules in living cells appears as the most reliable approach to investigate their structural properties and dynamic features. The macromolecular crowding and the stickiness of the cytoplasm, non-specific weak protein-protein interactions, as well as a myriad of potential interactors constitute parameters that can perturb protein structure and dynamics. Over the last years, several studies have highlighted the importance of exploring biomolecules directly in their intracellular environment [36]. However, a number of methodological and spectroscopic challenges still need to be addressed to make this approach broadly applicable to NMR, EPR and fluorescence, for instance. Elisabetta Mileo (CNRS, Marseille) presented how EPR spectroscopy can be used to investigate chaperones in actions directly inside cells [37]. In particular, Site-Directed Spin Labeling (SDSL)-EPR including Double Electron-Electron Resonance (DEER) techniques were used to get information on the structure and conformational dynamics of the NarJ chaperone [38], dedicated to the assembly of the membrane-bound respiratory nitrate reductase complex NarGHI, a molybdenum-iron containing metalloprotein in E. coli. Thanks to the development of an efficient method to deliver the paramagnetic-labeled-NarJ inside E. coli cells, E. Mileo and coworkers succeeded in performing intracellular EPR experiments at room temperature and at endogenous concentration $(30-40 \mu \mathrm{M})$, and they were able to follow the EPR signal in cells for more than 2 hours. These exciting results open up the perspective of achieving high resolution EPR studies of other chaperone proteins and their complexes in their native environment. 


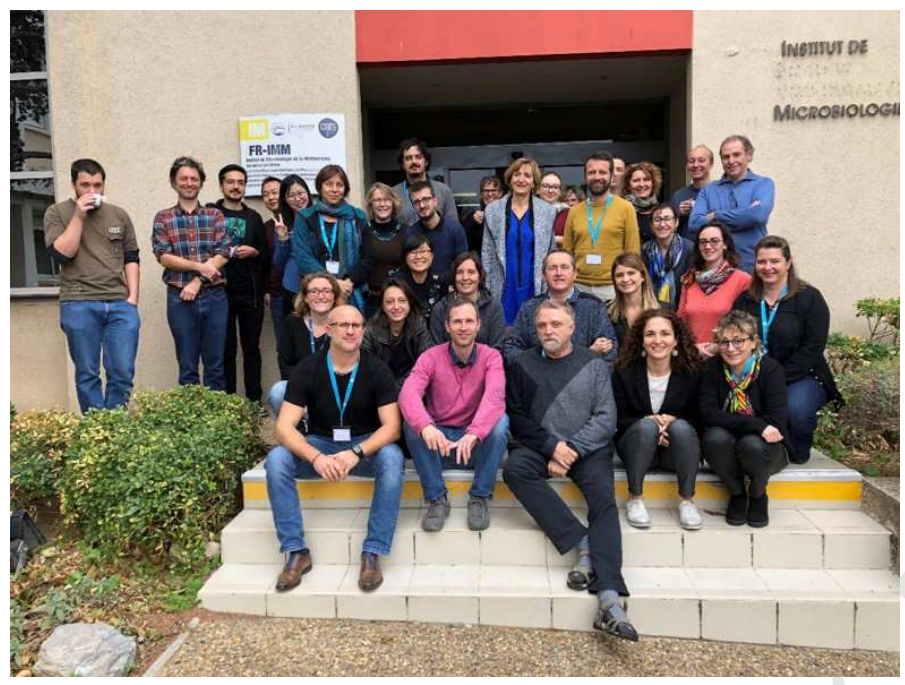

Figure 2: Group picture of the first French workshop on chaperone proteins and protein folding.

\section{Outlook}

This workshop demonstrated the different areas of biology and the growing number of technologies that the chaperone field now involves. The different talks presented at this conference highlighted the complexity hidden within these proteins and their variable functions. It is clear that emerging tools applied to different model organisms are necessary to understand better the ubiquitous role of chaperones.

There are still many outstanding questions in this field and we still have lots of work to do before solving all the mysteries of chaperones. Since this event was highly successful (Figure 2), we are looking forward to organize a second event. We are aware that there are many other scientists working in France in the field of chaperone proteins who were not present at this first workshop. Therefore, the next challenge for the second edition will be to gather new people to increase the French chaperone community.

\section{Acknowledgements}

We would like to thank the BIP laboratory for funding and support, Laure Azzopardi for her precious assistance in the organization of the workshop, all participants, and of course the members of our respective groups. 


\section{References}

[1] R.A. Laskey, B.M. Honda, A.D. Mills, J.T. Finch, Nucleosomes are assembled by an acidic protein which binds histones and transfers them to DNA, Nature. 275 (1978) 416-420.

[2] J. Ellis, Proteins as molecular chaperones, Nature. 328 (1987) 378-379. doi:10.1038/328378a0.

[3] P. Goloubinoff, A.S. Sassi, B. Fauvet, A. Barducci, P. De Los Rios, Chaperones convert the energy from ATP into the nonequilibrium stabilization of native proteins, Nat. Chem. Biol. 14 (2018) 388-395. doi:10.1038/s41589-018-0013-8.

[4] F.H. Schopf, M.M. Biebl, J. Buchner, The HSP90 chaperone machinery, Nat. Rev. Mol. Cell Biol. (2017). doi:10.1038/nrm.2017.20.

[5] L.J. Blair, J.J. Sabbagh, C.A. Dickey, Targeting Hsp90 and its co-chaperones to treat Alzheimer's disease, Expert Opin. Ther. Targets. 18 (2014) 1219-1232. doi:10.1517/14728222.2014.943185.

[6] M. von Bergen, P. Friedhoff, J. Biernat, J. Heberle, E.M. Mandelkow, E. Mandelkow, Assembly of tau protein into Alzheimer paired helical filaments depends on a local sequence motif ((306)VQIVYK(311)) forming beta structure, Proc. Natl. Acad. Sci. U. S. A. 97 (2000) 5129_ 5134.

[7] C. Schirmer, E. Lepvrier, L. Duchesne, O. Decaux, D. Thomas, C. Delamarche, C. Garnier, Hsp90 directly interacts, in vitro, with amyloid structures and modulates their assembly and disassembly, Biochim. Biophys. Acta. 1860 (2016) 2598-2609. doi:10.1016/j.bbagen.2016.07.033.

[8] J. Buchner, Bacterial Hsp90--desperately seeking clients, Mol. Microbiol. 76 (2010) 540-544. doi:10.1111/j.1365-2958.2010.07140.x.

[9] F.A. Honoré, V. Méjean, O. Genest, Hsp90 Is Essential under Heat Stress in the Bacterium Shewanella oneidensis, Cell Rep. 19 (2017) 680-687. doi:10.1016/j.celrep.2017.03.082.

[10]M.-P. Castanié-Cornet, N. Bruel, P. Genevaux, Chaperone networking facilitates protein targeting to the bacterial cytoplasmic membrane, Biochim. Biophys. Acta. 1843 (2014) 1442-1456. doi:10.1016/j.bbamcr.2013.11.007.

[11]P. Bordes, A.J. Sala, S. Ayala, P. Texier, N. Slama, A.-M. Cirinesi, V. Guillet, L. Mourey, P. Genevaux, Chaperone addiction of toxin-antitoxin systems, Nat. Commun. 7 (2016) 13339. doi:10.1038/ncomms13339.

[12]A.J. Sala, P. Bordes, S. Ayala, N. Slama, S. Tranier, M. Coddeville, A.-M. Cirinesi, M.-P. Castanié-Cornet, L. Mourey, P. Genevaux, Directed evolution of SecB chaperones toward toxinantitoxin systems, Proc. Natl. Acad. Sci. U. S. A. 114 (2017) 12584-12589. doi:10.1073/pnas.1710456114.

[13]G. Richarme, M. Mihoub, J. Dairou, L.C. Bui, T. Leger, A. Lamouri, Parkinsonism-associated protein DJ-1/Park7 is a major protein deglycase that repairs methylglyoxal- and glyoxal-glycated cysteine, arginine, and lysine residues, J. Biol. Chem. 290 (2015) 1885-1897. doi:10.1074/jbc.M114.597815.

[14]G. Richarme, C. Liu, M. Mihoub, J. Abdallah, T. Leger, N. Joly, J.-C. Liebart, U.V. Jurkunas, M. Nadal, P. Bouloc, J. Dairou, A. Lamouri, Guanine glycation repair by DJ-1/Park7 and its bacterial homologs, Science. 357 (2017) 208-211. doi:10.1126/science.aag1095.

[15]G. Richarme, E. Marguet, P. Forterre, S. Ishino, Y. Ishino, DJ-1 family Maillard deglycases prevent acrylamide formation, Biochem. Biophys. Res. Commun. 478 (2016) 1111-1116. doi:10.1016/j.bbrc.2016.08.077.

[16]J.C.A. Bardwell, U. Jakob, Conditional disorder in chaperone action, Trends Biochem. Sci. 37 (2012) 517-525. doi:10.1016/j.tibs.2012.08.006.

[17]B.J. Chang, A.B. Samal, J. Vlach, T.F. Fernandez, D. Brooke, P.E. Prevelige, J.S. Saad, Identification of the Calmodulin-Binding Domains of Fas Death Receptor, PloS One. 11 (2016) e0146493. doi:10.1371/journal.pone.0146493.

[18]D. Malinverni, A. Jost Lopez, P. De Los Rios, G. Hummer, A. Barducci, Modeling Hsp70/Hsp40 interaction by multi-scale molecular simulations and coevolutionary sequence analysis, ELife. 6 (2017). doi:10.7554/eLife.23471. 
[19]K. Macé, E. Giudice, S. Chat, R. Gillet, The structure of an elongation factor G-ribosome complex captured in the absence of inhibitors, Nucleic Acids Res. 46 (2018) 3211-3217. doi:10.1093/nar/gky081.

[20]D. Reichmann, U. Jakob, The roles of conditional disorder in redox proteins, Curr. Opin. Struct. Biol. 23 (2013) 436-442. doi:10.1016/j.sbi.2013.02.006.

[21]M. Ilbert, J. Horst, S. Ahrens, J. Winter, P.C.F. Graf, H. Lilie, U. Jakob, The redox-switch domain of Hsp33 functions as dual stress sensor, Nat. Struct. Mol. Biol. 14 (2007) 556-563. doi: $10.1038 / \mathrm{nsmb} 1244$.

[22]J. Winter, M. Ilbert, P.C.F. Graf, D. Ozcelik, U. Jakob, Bleach activates a redox-regulated chaperone by oxidative protein unfolding, Cell. 135 (2008) 691-701. doi:10.1016/j.cell.2008.09.024.

[23]B. Gontero, S.C. Maberly, An intrinsically disordered protein, CP12: jack of all trades and master of the Calvin cycle, Biochem. Soc. Trans. 40 (2012) 995-999. doi:10.1042/BST20120097.

[24]E. Graciet, P. Gans, N. Wedel, S. Lebreton, J.-M. Camadro, B. Gontero, The small protein CP12: a protein linker for supramolecular complex assembly, Biochemistry (Mosc.). 42 (2003) 81638170. doi:10.1021/bi034474x.

[25]J. Erales, S. Lignon, B. Gontero, CP12 from Chlamydomonas reinhardtii, a permanent specific "chaperone-like" protein of glyceraldehyde-3-phosphate dehydrogenase, J. Biol. Chem. 284 (2009) 12735-12744. doi:10.1074/jbc.M808254200.

[26]F. Mousson, F. Ochsenbein, C. Mann, The histone chaperone Asf1 at the crossroads of chromatin and DNA checkpoint pathways, Chromosoma. 116 (2007) 79-93. doi:10.1007/s00412-006-0087$\mathrm{Z}$.

[27]O.N. Lemaire, S. Bouillet, V. Méjean, C. Iobbi-Nivol, O. Genest, Chaperones in maturation of molybdoenzymes: Why specific is better than general?, Bioengineered. 8 (2017) 133-136. doi:10.1080/21655979.2016.1218579.

[28]D. Redelberger, O. Genest, D. Arabet, V. Méjean, M. Ilbert, C. Iobbi-Nivol, Quality control of a molybdoenzyme by the Lon protease, FEBS Lett. 587 (2013) 3935-3942. doi:10.1016/j.febslet.2013.10.045.

[29]S. Leimkühler, C. Iobbi-Nivol, Bacterial molybdoenzymes: old enzymes for new purposes, FEMS Microbiol. Rev. 40 (2016) 1-18. doi:10.1093/femsre/fuv043.

[30]P. De Los Rios, A. Barducci, Hsp70 chaperones are non-equilibrium machines that achieve ultraaffinity by energy consumption, ELife. 3 (2014) e02218.

[31]D. Malinverni, S. Marsili, A. Barducci, P. De Los Rios, Large-Scale Conformational Transitions and Dimerization Are Encoded in the Amino-Acid Sequences of Hsp70 Chaperones, PLoS Comput. Biol. 11 (2015) e1004262. doi:10.1371/journal.pcbi.1004262.

[32]E. Giudice, K. Macé, R. Gillet, Trans-translation exposed: understanding the structures and functions of tmRNA-SmpB, Front. Microbiol. 5 (2014) 113. doi:10.3389/fmicb.2014.00113.

[33]K. Macé, F. Demay, C. Guyomar, S. Georgeault, E. Giudice, R. Goude, A. Trautwetter, G. Ermel, C. Blanco, R. Gillet, A Genetic Tool to Quantify trans-Translation Activity in Vivo, J. Mol. Biol. 429 (2017) 3617-3625. doi:10.1016/j.jmb.2017.10.007.

[34]N. Richet, D. Liu, P. Legrand, C. Velours, A. Corpet, A. Gaubert, M. Bakail, G. Moal-Raisin, R. Guerois, C. Compper, A. Besle, B. Guichard, G. Almouzni, F. Ochsenbein, Structural insight into how the human helicase subunit MCM2 may act as a histone chaperone together with ASF1 at the replication fork, Nucleic Acids Res. 43 (2015) 1905-1917. doi:10.1093/nar/gkv021.

[35]Y. Jiao, K. Seeger, A. Lautrette, A. Gaubert, F. Mousson, R. Guerois, C. Mann, F. Ochsenbein, Surprising complexity of the Asf1 histone chaperone-Rad53 kinase interaction, Proc. Natl. Acad. Sci. U. S. A. 109 (2012) 2866-2871. doi:10.1073/pnas.1106023109.

[36]F.-X. Theillet, A. Binolfi, T. Frembgen-Kesner, K. Hingorani, M. Sarkar, C. Kyne, C. Li, P.B. Crowley, L. Gierasch, G.J. Pielak, A.H. Elcock, A. Gershenson, P. Selenko, Physicochemical properties of cells and their effects on intrinsically disordered proteins (IDPs), Chem. Rev. 114 (2014) 6661-6714. doi:10.1021/cr400695p.

[37]G. Karthikeyan, A. Bonucci, G. Casano, G. Gerbaud, S. Abel, V. Thomé, L. Kodjabachian, A. Magalon, B. Guigliarelli, V. Belle, O. Ouari, E. Mileo, A Bioresistant Nitroxide Spin Label for InCell EPR Spectroscopy: In Vitro and In Oocytes Protein Structural Dynamics Studies, Angew. Chem. Int. Ed Engl. 57 (2018) 1366-1370. doi:10.1002/anie.201710184. 
[38]M. Lorenzi, L. Sylvi, G. Gerbaud, E. Mileo, F. Halgand, A. Walburger, H. Vezin, V. Belle, B. Guigliarelli, A. Magalon, Conformational selection underlies recognition of a molybdoenzyme by its dedicated chaperone, PloS One. 7 (2012) e49523. doi:10.1371/journal.pone.0049523.

\section{Highlights}

- Meeting report for the First French Workshop on Chaperone Proteins, France, 2017.

- The meeting gathered scientists working in France on chaperone proteins.

- Themes were: breakthrough in chaperones, plasticity, specific chaperones, biophysical tools. 